\title{
Comparison between the Performances of a Bubble Pump Generator with Inlets Configurations
}

\author{
Bella Gurevich $^{1}$, Michael Jelinek ${ }^{2}$, Irene Borde ${ }^{2}$, Avi Levy ${ }^{2}$ \\ ${ }^{1}$ Shamoon College of Engineering, Mechanical Engineering Department \\ Jabotinsky 24, Ashdod, Israel \\ bellagu@sce.ac.il \\ ${ }^{2}$ Ben Gurion University of the Negev, Mechanical Engineering Department \\ Beer Sheva, 8410501 \\ mjelinek@bgu.ac.il; borde@bgu.ac.il; avi@bgu.ac.il
}

\begin{abstract}
The compressor or a mechanical pump of traditional refrigeration cycles is replaced by the bubble pump that circulates the binary working fluid in the diffusion absorption refrigeration (DAR) cycle. The bottleneck of the DAR cycle is the bubble pump, and its performance is determined by the amount of separated refrigerant that leaves the bubble pump. This work investigated the influence of the structure of the generator on the amount of desorbed refrigerant in a bubble pump system, with three parallel lifting tubes. Two modular experimental systems were designed to characterize the performance of three parallel bubble pump lift tubes with an environmentally-friendly binary solution of R134a-DMAC. The difference between the systems was in the design of the generator. One generator had three inlets and three outlets, and another one had a common inlet and three outlets. All other operating conditions remained the same. The results showed that an experimental system containing three inlets to the generator, produced more refrigerant than the system containing a common inlet.
\end{abstract}

Keywords: bubble pump, DAR cycle, multiple lift tubes, binary solution.

\section{Introduction}

Unlike conventional vapour compression and absorption refrigeration systems, diffusion absorption refrigeration (DAR) systems do not contain moving parts, and are heat driven. To circulate the working fluid without a mechanical pump or a compressor, the DAR system relies on the heat applied to a bubble pump, a heated tube (length $\mathrm{L}$ and diameter D) connecting two reservoirs; one situated higher than the other (Fig. 1). The binary liquid in the lower reservoir initially fills the tube to the same level as in the lower reservoir $(\mathrm{H})$. Some of the gas is then evaporated from the rich solution in the tube by heating the bottom of the bubble pump (generator), thus creating vapour bubbles that rise in the tube, carrying the liquid above them to the higher reservoir. The bulk density of the fluid in the tube is reduced relatively to that in the lower reservoir, thereby creating an overall buoyancy lift. The desorbed gaseous refrigerant is separated from the binary solution (poor solution) and flows to the condenser where heat is exchanged with the colder surrounding occurs. Auxiliary inert gas, ideally a non-condensable gas such as hydrogen or helium, is charged into the evaporator and the absorber. The presence of the inert gas reduces the partial pressure of the refrigerant, while preserving almost constant system pressure. This mixture of refrigerant and auxiliary inert gas flows through the evaporator tubes, where it absorbs heat from the cooling chamber. The now heated refrigerant and the inert gas flow to the absorber, where exothermic mixing occurs between the refrigerant and the poor solution. The inert gas is separated from this binary rich solution. Due to the motive head, this mixture flows to the lower reservoir of the bubble pump unit, where heat is applied.

The bottleneck of the DAR cycles is the bubble pump unit, and it is responsible for the low COP values that vary in range of 0.1-0.15. To achieve higher COP, the DAR system should not only desorb as much refrigerant in the separator, but also must utilize as minimum heat in the generator. These parameters define the bubble pump performance, however, are limited by the DAR unit configuration. 


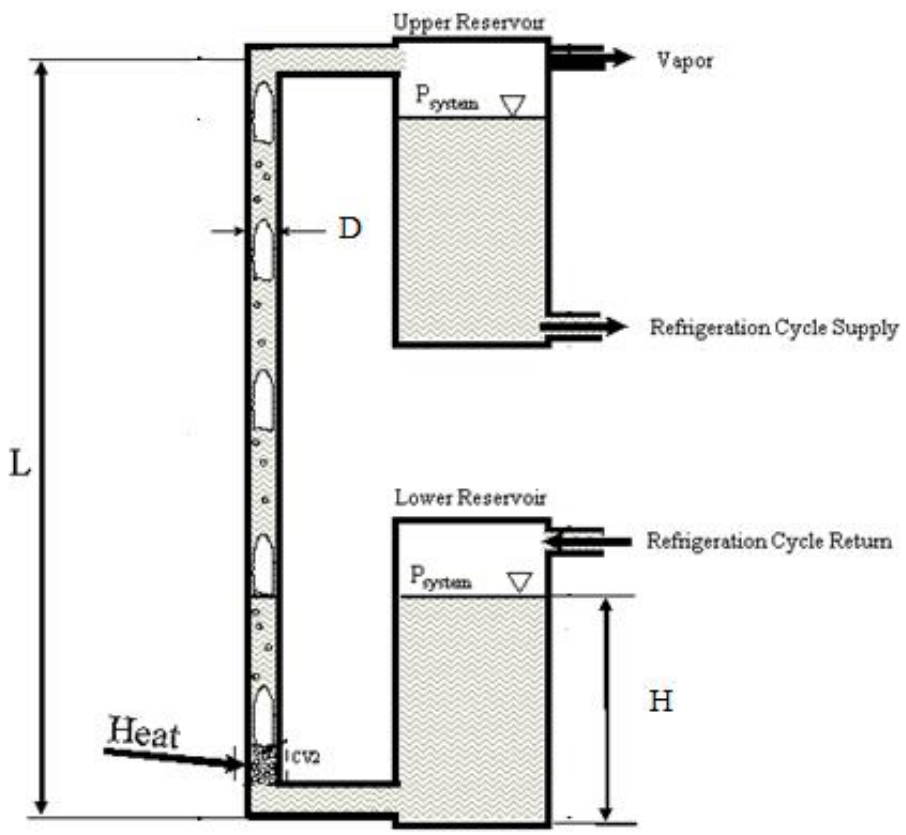

Fig. 1: Bubble pump schematic design.

Experimental studies by [1], [2] and [3] showed an increase of the vapour mass flow rate, with an increase in the supplied heat input until maximum value was reached for heat inputs, ranging between 150-200 Watts. Further increase in the heat input caused a decrease in the vapour mass flow rate. The results correspond well with the findings of [1] and [4], which had stated that the bubble pump did not operate most efficiently in the slug flow regime, in which vapour bubble diameter was approximately equal to that of the lifting tube. Slug flow patterns occur for diameters that are limited by the [5] correlation.

Experimental studies on the influence of the motive head on the bubble pump performance found that increasing the motive head caused an increase in the rich solution flow rate ([1], [4], [2], [3] and [6]).

Multiple lift tube configurations were experimentally investigated by [7], [8] and [9]. Their results indicated that an addition of lift tubes increased the pump's ability to handle larger heat loads and flow rates before the flow pattern changed from slug to annular.

The performance of a DAR system with three configurations of generator and bubble pump was studied numerically by [10]. It was found that the best performances were obtained by the second configuration (partially attached), when the heat was supplied directly into the rich solution and the bubble pump was attached to the returning poor solution. For equal heat input of $160 \mathrm{~W}$, the second configuration desorbed the highest amount of refrigerant.

None of the previously mentioned works tested the performance of a generator in the bubble pump unit operating with multiple lifting tubes.

\section{Experimental System}

Fig. 2 shows a layout of the experimental system of [9] and in Fig. 3, a layout of the present experimental system is show, with a variation in the structure of the generator.

In both of the systems, a standard single bubble pump configuration was replaced by a set of parallel bubble pumps operating with an environmentally friendly solution of R134a-DMAC (consideration in choosing the working fluids was based on work done by [11]). The design of the systems allow changes to the heat input, the motive head and enabled insertion of additional R134a to change the concentration of the solution. 


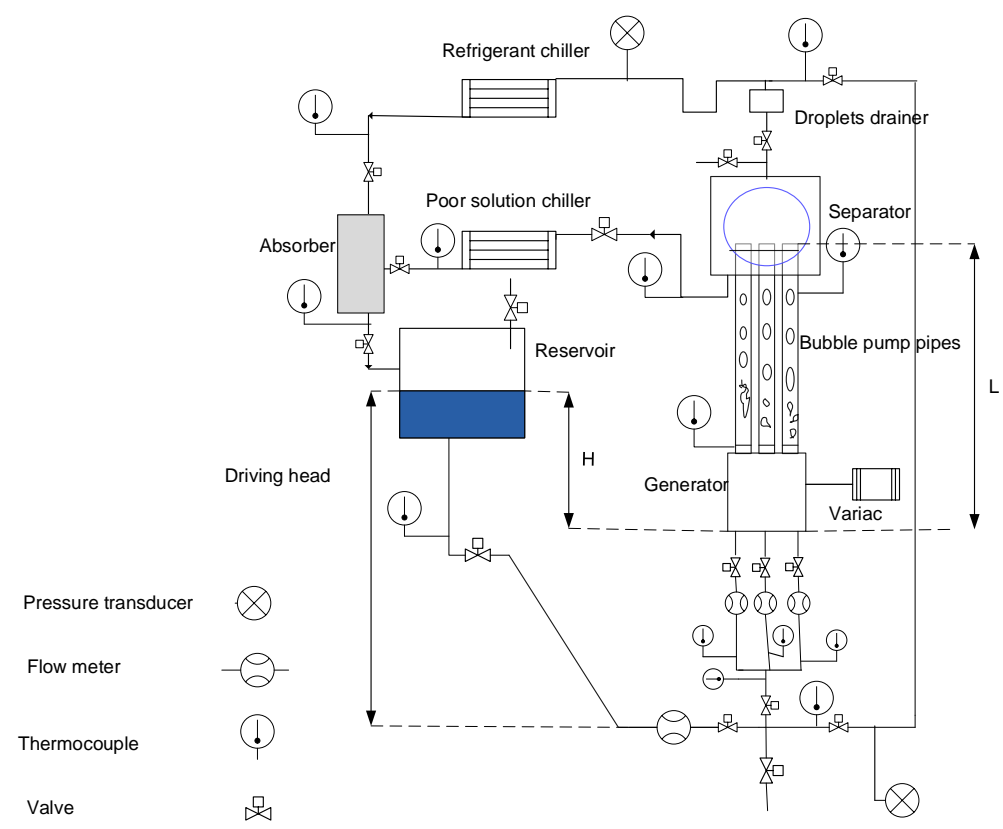

Fig. 2: [9] experimental system.

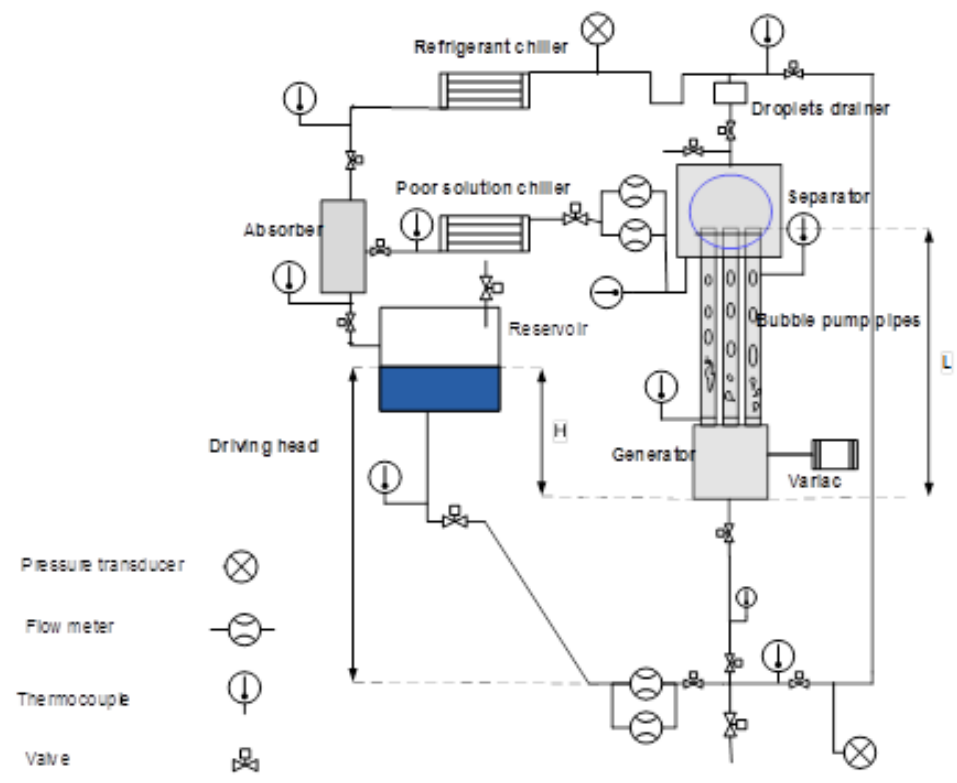

Fig. 3: Layout of the experimental system.

The experimental systems are lacking an evaporator, a condenser, and the inert gas, since they focus only on the performance of the bubble pump, and are not considered a complete diffusion absorption cooling system. Both systems comprise a reservoir, a heating unit (generator), a separation unit (separator), and an absorption unit, all of which are schematically illustrated in Fig.2 and Fig. 3. The main difference between the systems lays in the structure of the generator. In the experimental system designed by [9], the rich solution in the reservoir flows downwards to the manifold where valves could be opened or closed to direct the flow into one, using two or all three of the pipes. The three pipes are connected to the generator where the heat for refrigerant evaporation is supplied by a manually operated variac powered by an electrical DC current. Vertical flow is driven by the evaporated refrigerant bubbles, which carry the liquid solution up 
the lifting pipes to the separation unit. The new experimental system is not modular, since there is one rich solution pipe connected to the generator's entrance. Due to unique structure of the generator, the heated rich solution splits into three vertical lifting tubes. All other parts of the experimental systems remain the same. The separator's special double-outlet design enables upward flow of the gaseous refrigerant, and downward flow of the liquid poor solution. The liquid binary solution contains solvent and refrigerant that is not separated during evaporation (poor solution). The amount of separated refrigerant defines bubble pump performance. Both the hot refrigerant and the hot poor solution flow through bent copper tubes where they are cooled by heat exchange with the environment. The pipes for refrigerant and poor solution then merge into a single pipe, resulting in absorption of the refrigerant in the poor solution, a reaction accompanied by an exothermic effect, and further heat exchange with the environment. The combined solution of refrigerant and poor solution then flows back into the reservoir. The inclusion of metering devices in the system makes it possible to know the temperature, pressure, and flow rate at points of interest in real time.

\subsection{Experimental Results}

The aim of the experimental work was to test whether a change in the configuration of the generator influences the performance of the bubble pump, i.e. the amount of the desorbed refrigerant.

The modular design of the experimental system enables its operator to change relevant operational conditions, such as the concentration of the solution and the heat input in the generator. In order to obtain better comparison, all the results are normalized by the number of three pipes, i.e. the values of the separated refrigerant, and the required heating input are divided by the number of the lifting tubes. This normalization is performed in all of the following results. All of the experiments were performed for a motive head of $650 \mathrm{~mm}$, nominal concentration of $40 \%$ and were compared to experimental results of [9] with the same operating conditions when flow occurred in three pipes. These operating conditions were chosen because [9] experimental results indicated that at a motive head of $650 \mathrm{~mm}$ and nominal concentration of $40 \%$ for a case of three lifting tubes, more refrigerant was produced at the bubble pump. Error bars are not shown on the graphs because based on the experimental error analysis the errors were two orders smaller that the measured results.

A comparison between the amounts of the separated refrigerant of the two systems is shown in Fig. 4.

It is evident from the results that the old system having a generator configuration of three inlets desorbs more refrigerant. The old system described in the work of [9] desorbs maximum amounts of refrigerant at input power ranges of 150 to 200 Watts. Further increase in the input power results in a decrease of the refrigerant's mass flow rate. The new system shows a slightly monotonous increase in the amounts of the separated refrigerant with an increase in the system power input.

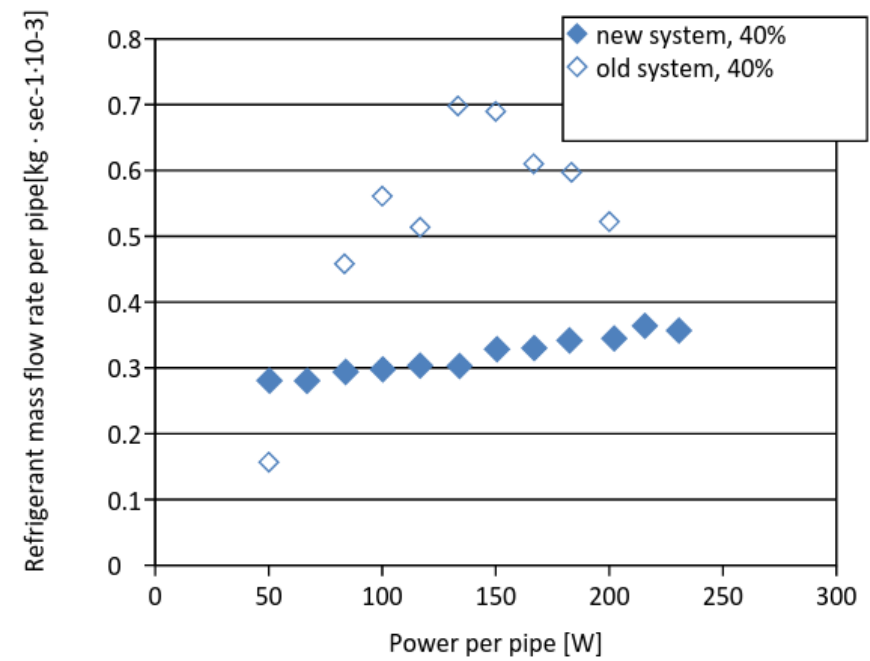

Fig. 4: Refrigerant mass flow rate vs. power input of the new and the old systems.

HTFF 107-4 
Fig. 5 shows the comparison between the temperatures of the reservoir for the old and the new systems. From the results, it can be seen that the new system having a generator with a common inlet has higher reservoir temperatures for various power inputs.

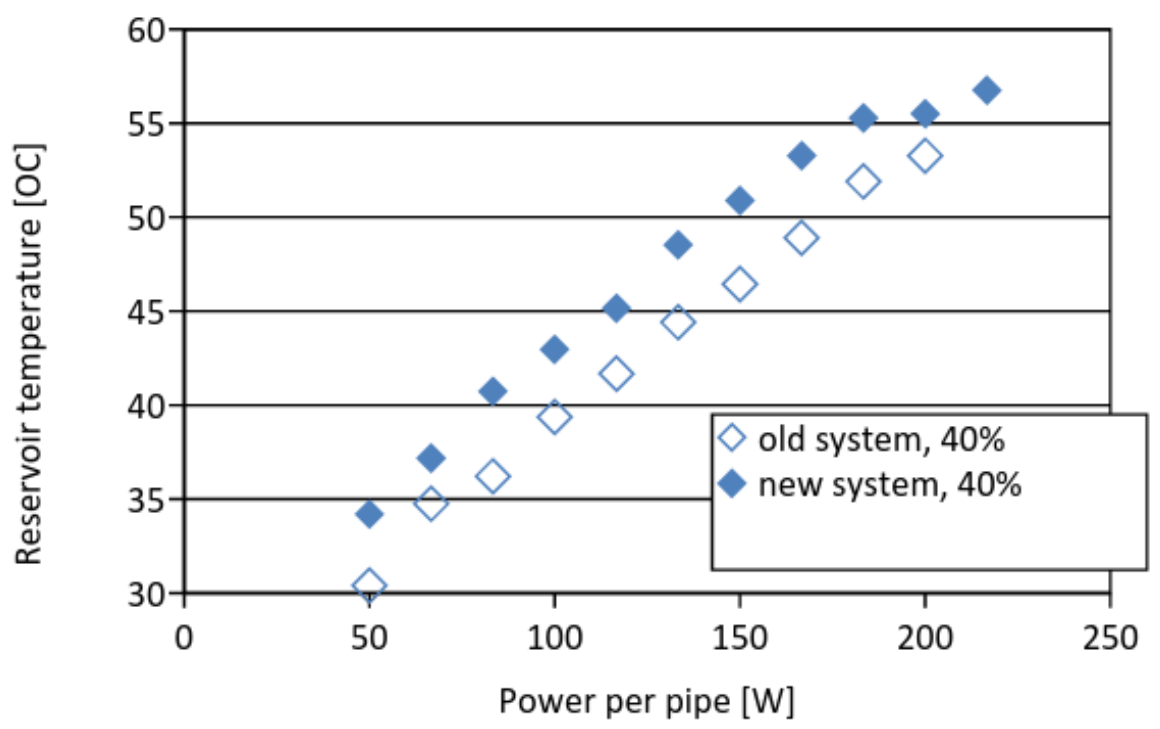

Fig. 5: Reservoir temperature vs. power input of the new and the old systems.

A comparison between generator temperatures as a function of the supplied heat input for the old and the new systems is presented in Fig. 6. It can be seen that the generator temperatures of the both systems are relatively equal.

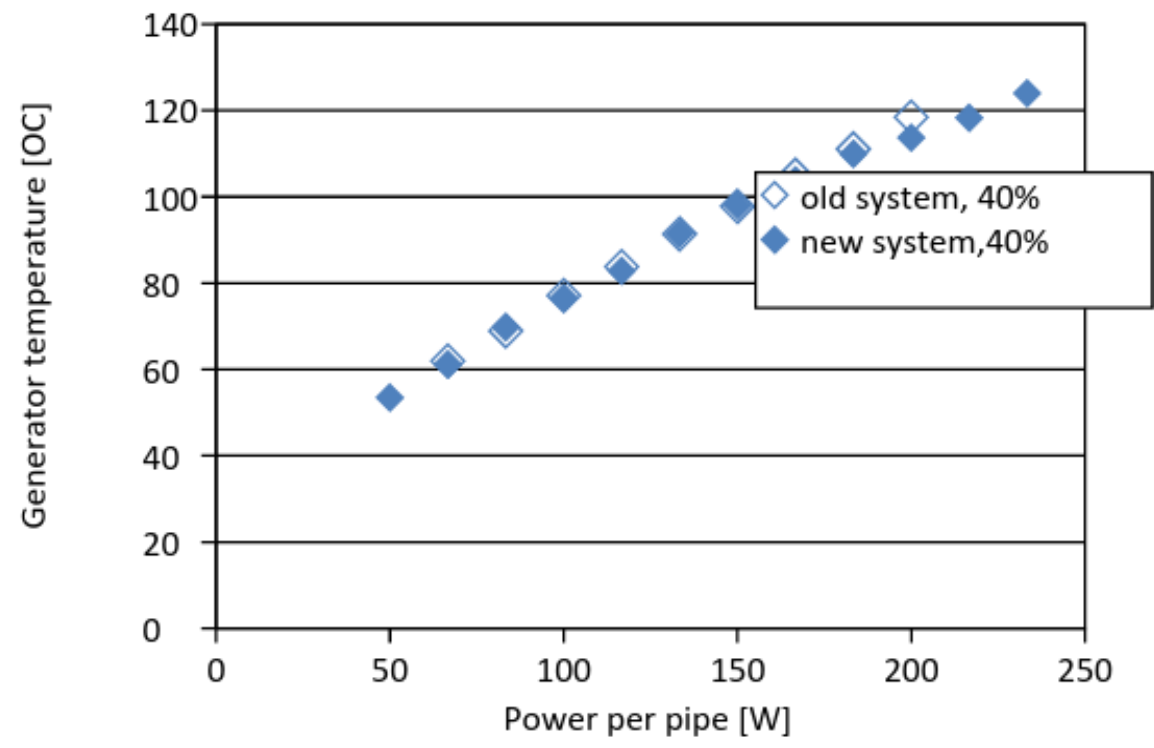

Fig. 6: Generator temperature vs. power input of the new and the old systems.

When comparing the system pressures of the two systems for the same heat inputs (Fig. 7), it can be seen that the system pressure remains pretty much the same for the two systems.

HTFF 107-5 


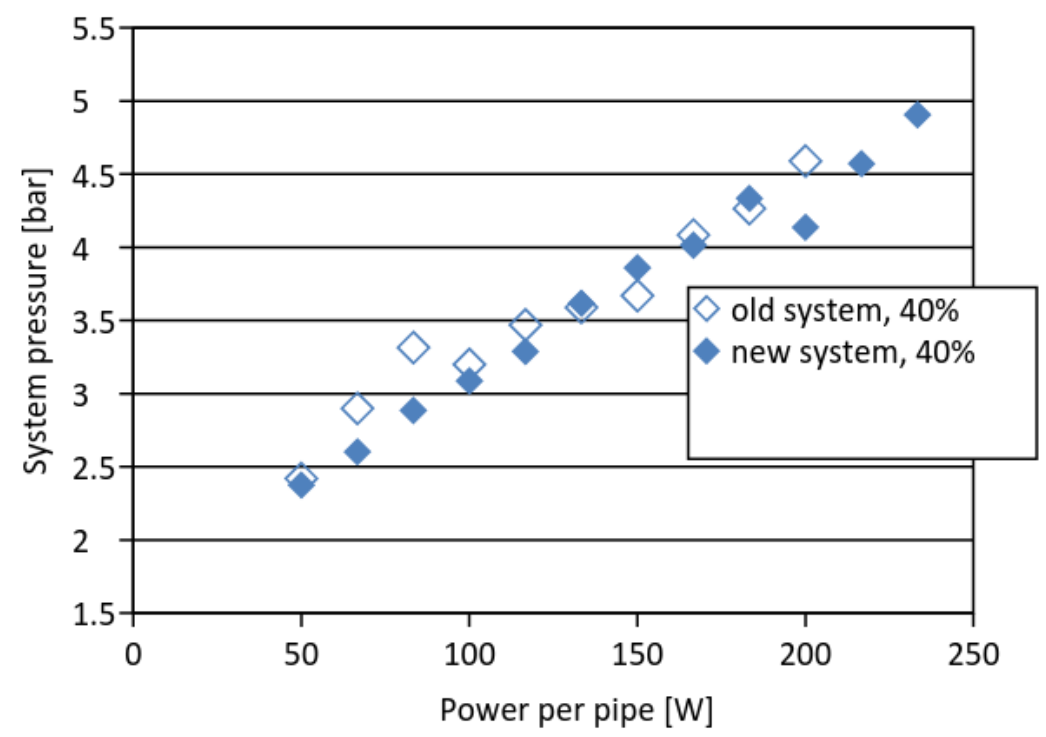

Fig. 7: System pressure vs. power input of the new and the old systems.

\section{Conclusions}

This work focused on the structure of the generator of the bubble pump having three parallel lifting tubes. Experimental results of a bubble pump unit having a generator with three inlets and three outlets were compared to experimental results of the same system having a generator with a common inlet. From the experimental results, it can be seen that the system consisting of a generator with three inlets produced much more refrigerant.

Comparison between the systems' pressure and the generator temperature showed no difference between the systems. The reservoir temperature was slightly higher for the new system having a common inlet generator structure.

One possible explanation of the difference in the results lays in the amounts of the rich solution. It can be inferred that a change in the configuration of the system from three parallel pipes entering the generator, to a single pipe reduced the amount of the rich solution in the system. The new configuration of the system contains less rich solution. Therefore, to reach the same system pressure, the solution at the reservoir is at a higher temperature. This can explain why less refrigerant is desorbed in comparison to the old system.

It can be stated the geometrical structure of the generator has a strong influence on the performance of the bubble pump unit.

\section{References}

[1] A. D. Delano, "Design analysis of the Einstein refrigeration cycle Robot Vision," Ph.D. dissertation, Georgia Institute of Technology, 1998.

[2] A. Sathe, "Experimental and Theoretical Studies on a Bubble Pump for a Diffusion-Absorption Refrigeration System," Master's thesis, Indian Institute of Technology Madras and University of Stuttgart, 2001.

[3] A. Koyfman, M. Jelinek, A. Levy and I. Borde, "An experimental investigation of bubble pump performance for diffusion absorption refrigeration system with organic working fluids," Applied Thermal Engineering, pp. 1881-1894, 2003.

[4] S. J. White, "Bubble pump design and performance," Master's thesis, Georgia Institute of Technology.

[5] D. Chisholm, Two-phase flow in pipelines and heat. New York: George Goodwin,1983.

[6] M. Pfaff, R. Saravanan, M. P Maiya and S. S Murthy, "Studies on bubble pump for a water-lithium bromide vapour absorption refrigerator," Int. J Refrigeration, vol. 21, no. 6, pp. 452-562, 1998.

[7] G. Vicatos and A. Bennett, "Multiple lift tube pumps boost refrigeration capacity in absorption plants," Journal of Energy in Southern Africa, vol. 18, no. 3, pp. 49-57, 2007.

HTFF 107-6 
[8] H. Monsef, M. Naghash Zadegan and K. Javaherdeh, "Design and Construction of a Low Capacity Pump-Less Absorption System," Mechanical Engineering Department, University of Guilan, Iran, 2012.

[9] B. Gurevich, M. Jelinek, A. Levy and I. Borde, "Performance of a set of parallel bubble pumps operating with a binary solution of R134a-DMAC," Applied Thermal Engineering, vol. 75, pp. 724-730, 2015.

[10] A. Zohar, M. Jelinek, A. Levy and I. Borde, (2009 "Performance of Diffusion Absorption Refrigeration Cycle with Organic Working Fluids," International Journal of Refrigeration, vol. 32, no. 6, pp. 1241-1246, 2009.

[11] I. Borde, M. Jelinek and N. C. Daltrophe, "Development of Advanced Absorption Systems Driven by Low Temperature Heat Sources," Int. J. Refrigeration, vol. 31, pp. 521-530, 1993. 